\title{
Les Interpretationes nominum Hebraicorum progenitorum Iesu Christi (ALC 62) : une oeuvre authentique d'Alcuin
}

Olivier Szerwiniack

\section{(2) OpenEdition \\ 1 Journals}

\section{Édition électronique}

URL : http://journals.openedition.org/abpo/1249

DOI : $10.4000 /$ abpo. 1249

ISBN : 978-2-7535-1495-9

ISSN : 2108-6443

\section{Éditeur}

Presses universitaires de Rennes

\section{Édition imprimée}

Date de publication : 20 septembre 2004

Pagination : 289-299

ISBN : 978-2-7535-0053-2

ISSN : 0399-0826

\section{Référence électronique}

Olivier Szerwiniack, «Les Interpretationes nominum Hebraicorum progenitorum lesu Christi (ALC 62) : une oeuvre authentique d'Alcuin », Annales de Bretagne et des Pays de l'Ouest [En ligne], 111-3 | 2004, mis en ligne le 20 septembre 2006, consulté le 02 mai 2019. URL : http://journals.openedition.org/ abpo/1249; DOI : 10.4000/abpo.1249 


\title{
Les Interpretationes nominum Hebraicorum progenitorum Iesu Christi (ALC 62) : une œuvre authentique d'Alcuin
}

\author{
Olivier SzERWINIACK \\ Maître de conférences de latin médiéval à l'Université de Picardie Jules-Verne \\ Centre d'Études sur le Moyen Âge et la Renaissance
}

Les Interpretationes nominum Hebraicorum progenitorum Iesu Christi sont considérées comme une œuvre authentique d'Alcuin dans le second tome de la Clauis Scriptorum Latinorum Medii Aeui-Auctores Galliae, où elles ont reçu le sigle ALC $62^{1}$. Pourtant Michael Gorman, dans un article récent, rejette cette œuvre comme inauthentique, puisqu'il la classe parmi trentesix œuvres faussement attribuées, "spuria ", que l'on peut selon lui exclure de manière certaine du canon des œuvres d'Alcuin ${ }^{2}$. Mon propos est donc de réexaminer le contenu de cette œuvre jusqu'ici très peu étudiée, afin de déterminer son authenticité et, brièvement, ses sources et ses parallèles.

\section{Présentation du texte}

Contrairement à la section de l'Évangile de Matthieu du Liber interpretationum Hebraicorum nominum de Jérôme ${ }^{3}$, qui donne dans l'ordre alphabétique la signification latine de la majorité des noms hébreux ${ }^{4}$ de l'Évangile de Matthieu et dont l'incipit est : Abraham pater uidens populum. Aminadab populus meus spontaneus..., notre texte traite seulement du Liber generationis Iesu Christi (Mt 1, 1-17) ${ }^{5}$, c'est-à-dire du début de l'Évangile de Matthieu, et les noms hébreux n'y sont pas classés par ordre alphabétique mais par ordre d'apparition dans la généalogie initiale du Christ : son incipit est : Abraham pater multarum. Isaac gaudium. Iacob subplantator... La

1. M.-H. Jullien et F. Perelman, Clauis..., t. II : Alcuinus, p. 468-469.

2. M. Gorman, « Alcuin before Migne », p. 129 et 127 : « It seems to me that we can confidently exclude the following items from the canon of Alcuin's works. "

3. Cf. Jérôme, Liber interpretationis nominum hebraicorum, p. 134-138.

4. Sur la question des noms hébreux et l'intérêt que ceux-ci ont suscité chez tous les exégètes médiévaux, cf. l'ouvrage fondamental de M. THIEL, Grundlagen und Gestalt der Hebräischkenntnisse ... et l'article de G. DAHAN, "Lexiques hébreu/latin... ».

5. Cf. Biblia sacra..., p. 1527. 
généalogie du Christ chez Matthieu se différencie de celle plus universelle, qui remonte à Adam et qu'on lit dans l'Évangile de Luc 3, 23-38 ${ }^{6}$, car elle est descendante, commence à Abraham seulement et se restreint presque toujours à la succession dynastique. Toutes ces différences expliquent que de David à Joseph les deux généalogies n'ont que deux noms en commun. En outre, pour respecter le symbolisme des chiffres (trois séries de deux fois sept noms), la généalogie de Matthieu omet entre Ioram et Ozias $(1,8)$ les noms des trois rois Ochozias, Ioas et Amasias, probablement parce qu'ils sont les descendants de l'impie Athalie qui voulut exterminer la lignée du Messie (2 R, 11, 1). Enfin, en plus des noms des pères, la généalogie mentionne aussi quelques noms de mères : Thamar, Rachab, Ruth et, sans la nommer, la femme d'Urie, c'est-à-dire Bethsabée ${ }^{7}$.

Notre texte est contenu dans plusieurs manuscrits ${ }^{8}$ cités par la Clauis, dont trois sont du IX ${ }^{\mathrm{e}}$ siècle :

- Trier, Stadtbibliothek, 23/122a et b, $\mathrm{f}^{\circ} 1-4 \mathrm{v}^{\circ}$ : malgré la restauration dont le manuscrit a fait l'objet, des trous et des taches d'humidité rendent le premier folio partiellement illisible. À la suite de notre texte, ce luxueux manuscrit des Évangiles en deux volumes du premier quart du IX $\mathrm{x}^{\mathrm{e}}$ siècle en provenance du monastère de Sainte-Marie aux Martyrs de Trèves contient plusieurs pièces liminaires et des tables des canons avant le texte des Évangiles proprement dit. Il a été présenté lors de plusieurs expositions du fait de ses nombreuses enluminures et initiales ornées dans un style " francoinsulaire ${ }^{9}$ ". Il est actuellement exposé dans le trésor de la bibliothèque (Schatzkammer) ${ }^{10}$.

\section{Ibidem, p. 1611-1612.}

7. La généalogie de Matthieu, contrairement à celle de Luc, souligne l'inscription de Jésus dans la généalogie davidique afin d'insister sur sa désignation messianique : cf. K. STENDAHL, "Quis et unde? An Analysis of Mt. 1-2".

8. Aux manuscrits cités par la Clavis, il faut ajouter le suivant : Kassel, Gesamthochschulbibliothek, Landesbibliothek und Murhardsche Bibliothek der Stadt, $2^{\circ}$ Ms. theol. $60, \mathrm{f}^{\circ} 3$ (seconde moitié $\mathrm{X}^{\mathrm{e}}$-début $\mathrm{Xl}{ }^{\mathrm{e}}$ siècle). Ce manuscrit, que nous n'avons pas pu examiner directement, semble ne comporter que la première partie du texte: cf. K. Wiedemann, Die Handschriften der Gesamthochschulbibliothek Kassel..., p. 85-88.

9. On trouvera sa description dans le catalogue de M. KEUFFER, Beschreibendes Verzeichnis der Handschriften ..., p. 25-28. Le manuscrit est daté d'environ 815-825 par B. FISCHER, Die lateinischen Evangelien... I. Varianten zu Matthäus, p. 26* (sigle Zt). Son écriture est postérieure à la mort de Charlemagne en 814 selon B. BIschoff, "Panorama der Handschriftenüberlieferung... ", p. 9, n. 17. Sur l'origine du manuscrit (Echternach, SaintMaximin de Trèves ou la France du Nord), son contenu et les descriptions successives dont il a fait l'objet, cf. R. LAUFNER, « Zur Herkunft des karolingischen Evangeliars... ». Plusieurs notices de catalogues d'exposition sont consacrées à ce manuscrit : cf. Kostbare Bücher und Dokumente..., p. 12-13; Trierer und Echternacher Handschriften ..., p. 28-32; G. FRANZ, Bibeln aus 1000 Jahren..., p. 28-29. Enfin, sur la restauration du manuscrit en 2000 : cf. B. I. HARAND, « Das Evangeliar von St. Maria ad Martyres... ». Je dois ces références au Pr. Dr Gunther Franz, directeur de la bibliothèque municipale de Trèves, auquel j'exprime toute ma gratitude.

10. Je remercie vivement le Dr Reiner Nolden, directeur des archives municipales de Trèves, de m'avoir permis d'examiner ce manuscrit précieux pendant deux jours. 
- Wolfenbüttel, Herz.-Aug.- Bibl., Weiss. 26 (4110), f ${ }^{\circ} 11-13$ : ce manuscrit des Évangiles de la seconde moitié du IX ${ }^{\mathrm{e}}$ siècle en provenance du monastère de Saint-Pierre de Wissembourg contient lui aussi plusieurs pièces liminaires, des tables des canons, puis notre texte avant les Évangiles proprement dits. Ces derniers sont abondamment glosés dans les marges ${ }^{11}$.

- München, B.S.B., lat. 14311, $\mathrm{f}^{\circ} 4 \mathrm{v}^{\circ}-8 \mathrm{v}^{\circ}$ : contrairement aux deux précédents, ce manuscrit de la seconde moitié du IX ${ }^{\mathrm{e}}$ siècle en provenance de Saint-Emmeram de Ratisbonne ne contient pas les Évangiles mais plusieurs textes exégétiques, dont un commentaire anonyme sur l'Évangile de Matthieu faussement attribué à Alcuin autrefois ${ }^{12}$.

Le titre Interpretationes nominum Hebraicorum progenitorum Iesu Christi est moderne. En effet, le texte s'intitule simplement Interpretatio nominum dans les manuscrits de Trèves et Wolfenbüttel et il est dépourvu de titre dans celui de Munich. Notre texte se compose de trois parties suivies de cinq distiques élégiaques (quatre dans le manuscrit de Munich) : on trouve d'abord une liste de noms hébreux munis de leur interprétation en latin, puis ces noms sont interprétés au sens mystique et enfin au sens moral, autrement dit l'auteur indique, souvent en s'appuyant sur des citations bibliques, comment ils s'appliquent au Christ dans la seconde partie, puis aux chrétiens dans la troisième.

Comme indiqué par la Clavis de Marie-Hélène Jullien et Françoise Perelman, l'édition la plus accessible de notre texte est celle du tome cent de la Patrologie latine, col. 725-734, qui reprend celle de Froben Forster de 1777. D'après les indications que ce dernier donne dans son avant-propos, il est manifeste qu'il édite le manuscrit de Munich, lat. 14311. Pourtant, son édition est illisible car il a inséré à la fois entre crochets dans le texte du manuscrit de Munich et en notes le texte de l'édition Duchesne ${ }^{13}$ de 1617, qui bizarrement reproduit dans son édition des œuvres d'Alcuin l'homélie 55 apocryphe du Pseudo-Bède ${ }^{14}$, souvent très proche, mais parfois aussi différente et qui doit donc être soigneusement distinguée de notre texte.

11. Ainsi par exemple au $\mathrm{f}^{\circ} 14 \mathrm{v}^{\circ}$, une glose marginale explique l'omission des trois noms entre Ioram et Ozias dans la généalogie initiale. Cf. la description complète du manuscrit par H. Butzmann, Kataloge der Herzog-August-Bibliothek Wolfenbüttel. Zehnter Band..., p. 134-136. Je remercie la bibliothèque de Wolfenbüttel de m'avoir procuré rapidement un microfilm et un tirage du texte.

12. Il s'agit du texte ALC Ps1, Anonymi in Mattheum, p. 512-513 de la Clavis. Ce long commentaire, qui occupe les folios 9 à $148 \mathrm{v}^{\circ}$ du manuscrit de Munich vient d'être édité d'après ce témoin unique par B. LOFSTEDT, Anonymi in Mattheum. D'après l'analyse de la langue et de l'orthographe, p. IX-Xv, B. Löfstedt estime que l'on ne peut donner de conclusion ferme sur l'origine du manuscrit car si certains indices indiquent une origine irlandaise, d'autres suggèrent une origine romane, sans pour autant être dirimants. On trouvera la description complète du manuscrit dans le Catalogus codicum ..., t. IV. 2, p. 157.

13. Sur cette édition et celle de Froben, cf. M. GoRMAN, "Alcuin before Migne ", p. 123124 et les planches 9-12, p. 118-121.

14. Cf. PL 94, col. 413-419, qui reprend l'édition du tome VII des Opera... omnia de Bède par Johannes HERWAGEN, Bâle, 1563. 
En effet, contrairement à ce dernier, cette homélie comprend notamment une introduction avant la liste d'interprétations et une conclusion à la fin de la troisième partie. En outre, elle ne comprend pas les distiques élégiaques. Il est donc nécessaire de procurer une nouvelle édition de ce texte, d'autant plus que l'édition du manuscrit de Munich par Forster n'est pas exempte d'approximations. Le nombre de pages qui nous est imparti ne nous permet malheureusement pas de publier en appendice une nouvelle édition critique du texte mais la collation des manuscrits de Munich $(M)$, Trèves (T), et Wolfenbüttel $(W)$ montre que $T$ et $W$ sont apparentés, sans toutefois être la copie l'un de l'autre, tandis que $M$ se distingue à son désavantage des deux autres sur plusieurs points. On lit par exemple dans $M$ contre $T$ et $W$ : "Bersabeae " pour Bethsabeae, "obes " pour oues, "Pooz" pour Booz, "inquinatis " pour iniquitatis, ou encore "Zoraobael " et "Zorobohel " pour Zorobabel. Le texte proposé par $T$ et $W$ semble donc meilleur, même si le manuscrit $T$, malgré son caractère luxueux, n'est pas exempt de fautes. On lit par exemple au $\mathrm{f}^{\circ} 77 \mathrm{v}^{\circ}$ en capitales rubriquées : Incipiunt capitulae secundum Marcum et au $\mathrm{f}^{\circ} 78 \mathrm{v}^{\circ}$ : Expliciunt capitulae.

Pour achever de présenter le texte, il faut signaler que les cinq distiques élégiaques ont été édités séparément par Ernst Dümmler dans les MGH $^{15}$, dans les pages consacrées aux poèmes d'Alcuin sous le $n^{\circ} 71.2$, bizarrement immédiatement après vingt vers consacrés aux quatre Évangiles qui ont reçu le $n^{\circ} 71.1^{16}$. Cette numérotation est inadéquate car les deux pièces versifiées n'ont rien en commun et elles sont totalement disjointes dans les deux manuscrits utilisés par Ernst Dümmler pour son édition. En effet, dans le manuscrit 23 de Trèves, nos distiques se trouvent au $f^{\circ} 4 v^{\circ}$ et les vingt autres vers aux folios $9 \mathrm{v}^{\circ}$ et 10 , tandis que dans le manuscrit de Wolfenbüttel, Wissembourg 26 , nos distiques sont au $\mathrm{f}^{\circ} 13$ et les vingt autres vers au folio $268 \mathrm{v}^{\circ}$.

\section{Une œuvre authentique}

Pour savoir si cette œuvre est authentique ou non, il faut partir des distiques puisque les trois premières parties ne comportent aucun nom d'auteur. $T$ et $W$ ont cinq distiques, mais $M$ seulement quatre, le cinquième distique étant absent. En voici l'édition critique suivie d'un essai de traduction $^{17}$ :

15. MGH Poet. I, p. 294.

16. Cf. la Clavis, ALC 11. 71.1, p. 90-91.

17. Pour rester compréhensible, j'ai parfois dû transposer certaines tournures latines en donnant un équivalent français. Il ne s'agit donc pas d'une traduction littérale. R. LAUFNER a traduit les distiques en allemand dans son article, « Zur Herkunft des karolingischen Evangeliars... ", p. 54. À ma connaissance, il s'agit de la première et unique traduction dans une langue moderne. La voici (la justification est celle de l'article) :

Nimm hin, König, nun das kleine Geschenk großer Liebe,

Das Alkuin, der Deine, sieh es, Dir darbringt.

Des Jahrhunderts Mächtige bringen Dir große Gaben aus Gaza, 
Suscipe, rex, paruum magni modo munus amoris, Quod tuus Albinus obtulit ecce tibi.

Magna ferunt saecli gazarum dona potentes, Fert mea pauperies ista minuta duo.

$\mathrm{Ne}$ uacua in sacris uenisset dextra diebus Ante piam faciem, rex uenerande, tuam,

Nomina sanctorum signaui sancta parentum Hebrea depromens ore, Latine, tuo.

Fer mea, carta mea, supplex, munuscula domno Corpore premodico uiscera magna gerens.

2. obtulit $M T$ : optulit $W$ 3. saecli $T W$ : secli $M$ 4. fert $M T W p c$ : fret $W a c$ 8. Hebrea $M W$ : Haebrea $T$ । । latine $M T p c W$ : latino Tac 9. munuscula $T p c W$ : munusculo Tac 9-10. hi uersus desunt in $M$.

Reçois, roi, en témoignage de sa grande affection ce modeste présent seulement

Que ton cher Albinus a apporté : le voici pour toi.

Les puissants du siècle apportent les cadeaux précieux de leurs trésors, Tandis que ma pauvreté n'apporte que ces deux petites pièces.

$5 \quad$ Pour éviter de paraître en ces jours sacrés les mains vides Devant toi, pieux et vénérable souverain,

Des saints ancêtres j'ai indiqué les saints noms Hébreux en les traduisant en latin ${ }^{18}$.

Porte, mon cher parchemin, en suppliant, mes maigres présents à mon seigneur :

10 Sous une enveloppe d'apparence modeste, tu transportes un contenu important ${ }^{19}$.

Meine Armut bringt her diese zwei Heller,

Auf daß keine leere Hand in den heiligen Tagen

Käm' vor Dein frommes Gesicht, ehrwürdiger König.

Die heil'gen hebräischen Namen der heiligen Väter

Hab' ich erläutert lateinisch, wie Deinem Mund ich entnahm'.

Bring [Fridugis] meinen Brief, meine Geschenkchen in Demut dem Herrn,

Entgegen dem dürftigen Leib tragend ein hochedles Herz.

18. Littéralement : " en les tirant de ta bouche, Latinus ». Par cette expression imagée, Alcuin veut dire qu'il a traduit en latin la signification des noms hébreux des ancêtres du Christ. Latine est le vocatif de Latinus car la scansion du pentamètre demande un $e$ bref. Il ne peut s'agir de l'adverbe latine, car, outre son $e$ long, celui-ci obligerait à comprendre qu'Alcuin tire de la bouche de Charlemagne les noms hébreux en latin, ce qui n'a guère de sens, à moins de supposer qu'Alcuin veut désigner Charles comme coauteur de l'ouvrage, comme il le fait dans les distiques initiaux du Dialogus de rhetorica et uirtutibus (ALC 39, éd. PL 101, col. 919-920). Cette dernière hypothèse n'est guère tenable car ce dialogue entre Alcuin et Charlemagne ne peut être comparé avec notre texte exégétique. Enfin, Charlemagne ne saurait être qualifié de Latin. Le nom Latinus au vocatif désigne bien plus probablement le roi du Latium (cf. Virgile, Énéide VII, 45), ancêtre éponyme des Latins, à moins qu'il ne s'agisse d'un personnage imaginaire incarnant les latinophones. Je remercie vivement $\mathrm{M}^{\mathrm{me}}$ Monique Goullet et MM. François Dolbeau et Édouard Jeauneau, qui m'ont aidé à comprendre ce vers difficile.

19. Pour replacer ces distiques dans le contexte des prologues poétiques d'Alcuin et plus généralement des auteurs carolingiens, qui développent tous plus ou moins la topique de l'humilité, cf. L. MunzI, « Prologhi poetici latini... ", p. 100-101. Je remercie François Dolbeau de m'avoir signalé cette référence et Luigi Munzi de m'en avoir envoyé un tiré à part. 
A priori, il ne fait aucun doute que ces vers sont d'Alcuin puisqu'ils citent son nom : "Albinus ". On pourrait toutefois arguer que le copiste de l'archétype des trois manuscrits a pu introduire le nom d'Alcuin sans que celui-ci en soit l'auteur véritable, car selon Michael Gorman des œuvres apocryphes furent attribuées à Alcuin dès le $\mathrm{IX}^{\mathrm{e}}$ siècle ${ }^{20}$. Cependant l'authenticité des cinq distiques est corroborée par la critique interne. En effet, les vers trois et surtout cinq et six rappellent les mots qu'Alcuin emploie dans sa lettre 261 accompagnant une Bible envoyée comme cadeau de Noël à Charlemagne par l'entremise de Fridugise alias Nathanael en $801^{21}$ : ne [...], aliis diuersa diuitiarum dona offerentibus, [...] uacuis manibus paruitatis meae missus ante faciem beatitudinis uestrae uenisset ${ }^{22}$. Ernst Dümmler a d'ailleurs émis l'hypothèse ${ }^{23}$ que ce cadeau de Noël pourrait être le manuscrit 23 de Trèves lui-même, mais Samuel Berger ${ }^{24}$ et Bonifatius Fischer ${ }^{25}$ ont montré que cela est impossible car Alcuin parle dans sa lettre d'une Bible entière et non des seuls Évangiles.

On retrouve également plusieurs des mots employés ici dans la préface en douze distiques élégiaques du livre second de la Vie de saint Willibrord écrite par Alcuin ${ }^{26}$ : ecce, uenerande (v. 1), minuta (v. 8), minuta duo (v. 15), supplex (v. 16), enfin gazas (v. 23). Dans cette préface comme dans nos distiques, Alcuin développe le thème de l'humilité et qualifie ses deux opuscules de piécettes "minuta ", en référence à l'épisode évangélique des deux pièces de monnaie qu'une pauvre veuve donna en offrande au Temple de Jérusalem $^{27}$. Enfin dans le cinquième distique Alcuin personnifie le par-

20. Cf. M. Gorman, "Alcuin before Migne », p. $101:$ " a tradition of attributing works to Alcuin that dates back to the ninth century. " et p. 124 : "From the ninth century onwards it was common practice to attribute all kinds of works to Alcuin. "

21. Cf. Alc. Ep. 262 (lettre d'instructions à Fridugise).

22. Alc. Ep. 261, p. 418-419.

23. Cf. E. DummLer, ibidem, p. 419, n. 1. Cette hypothèse est reprise par R. LAUfNER, «Zur Herkunft des karolingischen Evangeliars... ", p. 54-55 et dans les notices des catalogues d'exposition cités dans la note 9 .

24. Cf. S. BERGER, Histoire de la Vulgate..., p. 194, n. 3 : " Jaffé et après lui M. Dümmler ont cru, bien à tort, reconnaître dans le manuscrit 23 de Trèves le volume offert à Charlemagne en 801, de la part d'Alcuin. C'est également par erreur que l'on a donné ce manuscrit comme venant de Prüm. "On notera que S. Berger admet l'authenticité du poème, ibidem : "Il est bien l'œuvre de l'abbé de Saint-Martin de Tours, mais il est simplement destiné à présenter à l'empereur une "Interprétation des noms hébraïques". "

25. Cf. B. Fischer, "Bibeltext und Bibelreform... ", p. 106-107, n. 10, idem, "Die AlkuinBibeln », p. 224 : «Was Dümmler als zweiten Teil des Gedichtes gedruckt hat, ist eine echte Widmung von Alkuin an Karl, die in der Formulierung an den oben behandelten Brief 261 anklingt. Aber sie gehört trotzdem nicht zu diesem Brief oder zu dem Pandekten, den Alkuin auf Weihnachten 801 überreichen ließ, sondern zu einer kleinem Schrift in der Alkuin die Namen der Genealogie Christi am Anfang des Matthäus-Evangeliums nach dem wörtlichen, allegorischen und moralischen Sinn deutete. Das zeigt eindeutig sowohl die handschriftliche Überlieferung wie der Wortlaut der Verse selber. "

26. Éd. E. DummLER, MGH Poet. 1, p. 208-209.

27. Cf. Lc 21, 1-2 : Respiciens autem uidit eos qui mittebant munera sua in gazofilacium diuites. Vidit autem et quamdam uiduam pauperculam mittentem aera minuta duo et Mc 12, 41-42 : Cum uenisset autem una uidua pauper, misit duo minuta quod est quadrans et 
chemin sur lequel ses deux opuscules sont écrits comme il personnifie certaines de ses lettres ${ }^{28}$. L'authenticité de ces vers est donc certaine.

Il est indubitable d'après les vers quatre, sept et huit qu'ils accompagnent les opuscules précédents qui interprètent en latin la signification des noms hébreux des ancêtres du Christ. Toutefois, comme on l'a déjà dit, le texte qui précède les distiques se compose de trois parties : on trouve d'abord une liste de noms hébreux munis de leur interprétation littérale en latin, puis ces noms sont interprétés au sens mystique et enfin au sens moral. Pourquoi Alcuin parle-t-il donc de deux et non trois petites pièces? Le chiffre deux n'est probablement pas à prendre au pied de la lettre : il faut y voir une référence aux deux pièces de monnaie de la veuve plutôt que le nombre exact de textes qui précèdent les distiques. Dans le premier vers en effet Alcuin parle d'un seul présent et non de trois : il considère donc les trois parties et les distiques comme un tout. En tout état de cause, les trois parties ne sont pas de même nature : la première est une simple liste, alors que les deux autres sont illustrées par des citations bibliques. Cette bipartition est confirmée par l'étude des sources. En effet, la première partie a une source différente des deux autres qui sont inspirées d'un ouvrage d'Ailerán. Il est donc également possible de diviser l'ouvrage d'Alcuin en deux grandes parties, la seconde comportant deux sous-parties.

\section{Le travail d'Alcuin : sources et parallèles}

Alcuin donne l'interprétation littérale, puis mystique ou spirituelle et enfin morale de tous les noms de personnes cités dans la généalogie du Christ au début de l'Évangile de Matthieu. À ces noms il en ajoute deux : celui de Bethsabée, la femme d'Urie, dont Matthieu ne donne pas le nom et celui d'Amasias, l'un des trois rois absents de la généalogie initiale. Dans l'immense majorité des cas, les interprétations littérales des noms hébreux ont pour source ultime le Liber interpretationum nominum Hebraicorum de Jérôme. Toutefois, contrairement à ce qu'écrit Franz Brunhölzl dans son Histoire de la littérature latine du Moyen Âge ${ }^{29}$, il est probable qu'Alcuin n'a pas utilisé Jérôme directement pour écrire sa première partie. En effet le Liber questionum in Euangeliis présente une liste semblable avec les deux interprétations supplémentaires des noms de Bethsabée et Amasias. Certes les deux seuls manuscrits du Liber questionum in Euangeliis qui contiennent aujourd'hui cette liste, Orléans, Bibliothèque municipale, 65 (62) et Paris, Bibliothèque nationale de France, lat. $2384^{30}$, datent de la première

conuocans discipulos suos, ait illis : "amen, dico uobis quoniam uidua haec pauper plus omnibus misit qui miserunt in gazofilacium. "Sur le développement de ce thème chez Alcuin et Raban Maur, cf. M. PERRIN, "La pauvre veuve... ». Je remercie M. Perrin de m'avoir signalé son article.

28. Cf. sur ce point le bel article de C. VeYrard-Cosme, "Saint Jérôme... ", p. 328.

29. F. BRunholzL, Histoire de la littérature latine..., p. 36-37.

30. Ce manuscrit contient également une liste d'interprétations de noms hébreux attribuée à Bède : cf. notre article " Bède et les interprétations... ", p. 118 sqq. 
moitié du IX ${ }^{\mathrm{e}}$ siècle et ne semblent donc pas pouvoir être la source d'Alcuin, mais Jean Rittmueller vient de montrer dans l'introduction détaillée de son édition récente que le Liber questionum in Euangeliis est un commentaire hiberno-latin anonyme écrit dans la première moitié du viII ${ }^{\mathrm{e}}$ siècle (ca. 725), peut-être à Bangor ${ }^{31}$. Selon elle, le Liber adapte le commentaire sur Matthieu de Frigulus (floruit circa 700) encore inédit et connu par l'unique manuscrit Halle, Universitäts- und Landesbibliothek Sachsen-Anhalt, Quedlinburg 127 de la seconde moitié du IX ${ }^{\mathrm{e}}$ siècle.

Lorsque le Liber questionum in Euangeliis n'indique qu'une seule interprétation littérale, comme Vrias lux mea Dei, Alcuin la reprend sans la modifier. En revanche, lorsqu'il propose deux interprétations, voire davantage, Alcuin n'en conserve qu'une, sauf exception : Zara oriens aut coccineus devient Zara oriens. Toutefois pour l'interprétation d'Amasias, le Liber questionum in Euangeliis présente un saut du même au même et propose donc à sa place celle d'Ozias, contrairement au manuscrit de Frigulus qui offre une interprétation double Amasias robustus Domini siue populus eleuatus, dont la seconde partie se retrouve dans $M$ : Amasias populus eleuatus (electus $T W$ ). Il semble donc qu'Alcuin a eu accès soit à un manuscrit plus complet du Liber questionum, soit à un manuscrit plus ancien de Frigulus. La publication prochaine dans le Corpus Christianorum de l'édition du Commentarium in Euangelium secundum Mattheum de Frigulus par Anthony Forte devrait permettre de répondre à cette question.

En outre, deux autres manuscrits présentent une liste d'interprétations littérales comparable à celles d'Alcuin et du Liber questionum in Euangeliis : il s'agit des manuscrits Paris, Bibliothèque nationale de France, lat. 1841, $\mathrm{f}^{\circ} 159 \mathrm{v}^{\circ}-160$ (milieu du IX ${ }^{\mathrm{e}}$ siècle) et Munich, Bayerische Staatsbibliothek., lat. $6235, \mathrm{f}^{\circ} 65 \mathrm{v}^{\circ}-66$ (deuxième moitié du Ix ${ }^{\mathrm{e}}$ siècle). Ces deux manuscrits exégétiques contiennent plusieurs pièces qui expliquent les Évangiles, certaines d'origine probablement irlandaise ${ }^{32}$. Faute de pouvoir éditer en parallèle les trois listes afin de montrer tous les apparentements entre elles, je me limiterai à quelques observations. Les deux manuscrits Paris 1841 et Munich 6235 présentent pour Amasias l'interprétation suivante : Amasias robustus Domini siue eleuatus. Celle-ci est plus proche de la version de Frigulus que de celle d'Alcuin. La collation intégrale de la liste des deux manuscrits avec celles du Liber questionum in Euangeliis et d'Alcuin montre que dans presque tous les cas, ses interprétations sont plus proches du Liber questionum que d'Alcuin. En revanche, son interprétation Maria inluminatrix uel stilla maris est bien plus proche de celle d'Alcuin, Maria inluminatrix siue stella maris, que du Liber questionum, qui donne la version suivante : Maria ut plerique interpretantur inluminant me isti uel inluminatrix uel smirna maris aut amarum mare. Sermone autem Syro domina dicitur.

31. Liber questionum in Euangeliis, éd. J. RITTMUELLER, p. 30*. L'introduction particulièrement détaillée de J. Rittmueller remplace désormais la notice ALC 65 de la Clavis.

32. Pour une description plus complète de ces deux manuscrits, cf. E. Muluins et O. SZERWINIACK, "Incipit interpretatio paucorum... ". 
Pour la première partie, Alcuin semble donc avoir travaillé sur une version plus ancienne et légèrement différente du Liber questionum in Euangeliis, dont les deux manuscrits de Paris et Munich conservent une trace.

Si pour la première partie le travail d'Alcuin a consisté à reproduire en l'abrégeant une version antérieure de la liste d'interprétations de noms hébreux du Liber questionum, il est en revanche beaucoup plus important pour les deux parties suivantes. Alcuin y reprend en effet chaque nom et montre, parfois en s'appuyant sur une citation biblique, que sa signification s'applique aussi au Christ (interprétation mystique ou spirituelle) et aux chrétiens (interprétation morale). On notera toutefois que certains noms ne sont pas repris : les noms des mères Thamar, Rachab, Ruth et Bethsabée, ainsi que celui d'Amasias, sont absents de la troisième partie et le nom d'Urie ne figure ni dans la troisième ni dans la seconde.

Pour ces deux dernières parties, Alcuin a profondément remanié l'ouvrage de l'irlandais Ailerán, Interpretatio mystica et moralis progenitorum Domini Iesu Christi, qui se compose lui-même de deux parties ${ }^{33}$. Les deux manuscrits les plus anciens de cet ouvrage datent du IX ${ }^{\mathrm{e}}$ siècle : l'un, incomplet, Sankt-Gallen, Stiftsbibliothek 433, porte le nom d'Aileranus Scottus, mais l'autre, pourtant complet, Karlsruhe, Badische Landesbibliothek, Augiensis CCXLIX, ne présente ni titre ni nom d'auteur. L'attribution à Ailerán est toutefois confirmée par Sedulius Scottus, qui dans son Collectaneum in Matthaeum reprend sous une forme très abrégée le texte de l'Interpretatio mystica et moralis progenitorum Domini Iesu Christi, qu'il attribue à sanctus Aileranus Scottorum sapientissimus ${ }^{34}$. Le personnage d'Ailerán n'en demeure pas moins très mal connu : il semble avoir vécu au monastère de Clonard en Irlande et être mort en $665^{35}$.

Les deux parties d'Ailerán sont beaucoup plus longues et détaillées que celles d'Alcuin qui les a considérablement abrégées, même s'il arrive que ce dernier allonge certaines interprétations d'Ailerán. En outre, Alcuin n'a pas hésité à modifier parfois presque totalement certaines interprétations et à en ajouter d'autres. Ainsi Alcuin a complété Ailerán chez qui les noms des mères Thamar, Rachab, Ruth et Bethsabée, ainsi que celui d'Amasias, sont absents de son Interpretatio mystica. Il serait trop long d'entrer dans le détail, mais hormis quelques cas de reprise mot à mot, il y a un véritable travail de réécriture par Alcuin.

33. Éd. PL 80, col. 327-342 à compléter par PL Supplementum IV, col. 1612-1623. L'édition d'A. BREEn, Ailerani interpretatio..., qui édite à la fois le texte complet d'Ailerán (p. 17-35), la recension abrégée de Sedulius Scottus (p. 36-43) et deux autres recensions contenues dans les manuscrits Vienne, Österreichische Nationalbibliothek, lat. 940, vIII - IX ${ }^{\mathrm{e}}$ siècle, $\mathrm{f}^{\circ} 19-21 \mathrm{r}^{\circ}$ (p. 72-75) et Londres, British Library, Add. ms. 19835, xII $^{\mathrm{e}}$ siècle, f ${ }^{\circ} 46 \mathrm{v}^{\circ}-48$ (p. 7880), est presque introuvable en France, hormis l'exemplaire de l'I.R.H.T. Elle a fait l'objet d'un compte rendu par J. RitTMUELLER, "The New edition... " et de remarques critiques par M. GORMAN, «The Myth... ", p. 70-72.

34. Cf. éd. B. LOFSTEDT, Anonymi in Mattheum, p. 34 et éd. A. BREEN, Ailerani interpretatio..., p. 36.

35. Cf. I. MACHIELSEN, Clauis patristica pseudepigraphorum..., vol. II A, p. 393, n ${ }^{\circ} 1735$, A. Breen, Ailerani interpretatio..., p. 1-2 et N. Stalmans, Saints d'Irlande..., p. 63-64. 
Reste la question du rapport d'Alcuin avec l'homélie 55 du Pseudo-Bède : outre les différences déjà signalées plus haut, l'homélie inclut les noms des mères Thamar, Rachab, Ruth et Bethsabée, ainsi que le nom d'Urie dans l'interprétation morale, alors qu'ils en sont absents chez Alcuin. En revanche, le nom d'Amasias manque dans la liste initiale d'interprétations littérales de l'homélie. A priori, il n'est pas impossible qu'Alcuin ait connue l'homélie 55 du Pseudo-Bède et qu'il l'ait remaniée, mais il semble plus vraisemblable que l'auteur de l'homélie soit postérieur à Alcuin et qu'il ait voulu compléter le texte de celui-ci en ajoutant les noms des mères et d'Urie absents de sa troisième partie. Seule la recherche exhaustive des manuscrits $^{36}$ de l'homélie 55 permettrait de préciser l'ordre chronologique entre ce texte et celui d'Alcuin. Pour l'heure ce point reste sujet à discussion.

En conclusion, il me semble indéniable qu'Alcuin a rédigé un petit traité en trois parties sur les noms hébreux cités dans la généalogie du Christ au début de l'Évangile de Matthieu pour faire un cadeau à Charlemagne à l'occasion d'une fête de Pâques ou de Noël. Pour cela, comme tous les auteurs médiévaux, Alcuin a remanié des sources antérieures : il a abrégé une ancienne version du Liber questionum in Euangeliis pour la première partie et il a réécrit presque entièrement l'Interpretatio mystica et moralis progenitorum Domini Iesu Christi d'Ailerán pour les deux autres.

Ce traité d'Alcuin s'inscrit dans un ensemble de textes qui témoignent de l'intérêt des milieux irlandais et northumbriens pour les interprétations des noms hébreux ${ }^{37}$. Il pourrait donc, comme le suggère Donald A. Bullough dans son livre posthume ${ }^{38}$, dater des années 790-793, lorsqu'Alcuin, de retour à York, put de nouveau consulter les manuscrits irlandais et northumbriens qui se trouvaient dans la bibliothèque de l'archevêché.

36. Le manuscrit fragmentaire Darmstadt, Hessische Landes- und Hochschulbibliothek 2766 (XIV $^{\mathrm{e}}$ siècle), $\mathrm{f}^{\circ} 16 \mathrm{r}^{\circ}-\mathrm{v}^{\circ}$, cité par la Clavis, p. 469, contient en fait le début de l'homélie 55. Je remercie I. Bröning et le Dr Uhlemann de m'avoir gracieusement communiqué une copie du folio $16 \mathrm{r}^{\circ}-\mathrm{v}^{\circ}$. Sur l'ensemble dont fait partie cette homélie, cf. Dom J. LECLERCQ, " Le III ${ }^{\mathrm{e}}$ livre des Homélies... ".

37. Cf. nos deux articles " Des recueils d'interprétations... " et " Bède et les interprétations... ».

38. D. A. Bullough, Alcuin : Achievement and Reputation, p. 274. 


\section{RESUME}

Après une rapide présentation de l'ouvrage connu aujourd'hui sous le nom d'Interpretationes nominum Hebraicorum progenitorum Iesu Christi (ALC 62), son authenticité, récemment contestée par Michael Gorman, est démontrée, puis les sources et le travail d'Alcuin sont brièvement analysés.

\section{ABSTRACT}

In answer to M. Gorman, who in a recent paper has contested the authenticity of the text known today as Interpretationes nominum Hebraicorum progenitorum Iesu Christi (ALC 62), this paper presents its content, proves its authenticity and briefly analyses its sources and Alcuin's work upon them. 
